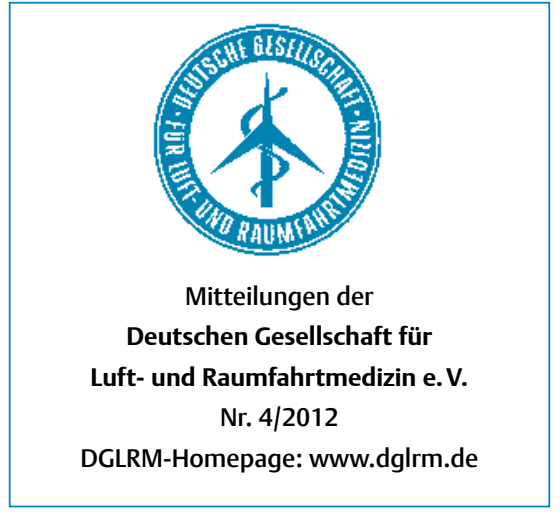

Geschäftsstelle der DGLRM

Frau Doris Mittelstädt - DLR

Linder Höhe

51170 Köln

E-Mail: geschaeftsstelle@dglrm.de

Sprechzeiten: mittwochs 14:00-16:00 Uhr

Tel.: 0176/56242876

\section{Verantwortlich für den Inhalt} der DGLRM-Seiten

Deutsche Gesellschaft für Luft- und Raumfahrtmedizin e.V. (DGLRM) Dr. Claudia Stern, Präsidentin, DLR, 51170 Köln, claudia.stern@dlr.de

Redaktionsschluss: 16.07.2012

Bankverbindung

Deutsche Bank Hamburg,

Konto-Nr.: 3889482, BLZ: 20070000

IBAN: DE 63200700240388948200 ,

SWIFT/BIC: DEUTDEDBHAM

Änderungen für die Mitgliederkartei bitte an: Dr. Martin Trammer, DLR,

51170 Köln, martin.trammer@dlr.de

\section{Wir trauern um}

Dr. Heinz Hofherr, der am 27.06.2012 im Alter von 71 Jahren verstarb.

\section{Wir gratulieren}

- 65 Jahre, Prof. Dr. Reinhard G. Matschke, Hannover, 07.07.1947

- 65 Jahre, Dr. Hans-Werner Teichmüller, Krefeld, 27.07.1947

- 105 Jahre, Prof. Dr. Alfred Johannes Koch, Münster, 23.06.1907

Wir begrüßen als neue Mitglieder

Anne Weißleder, Bad Blankenburg

Rebekka Deißer, Görmin

\section{Sehr geehrte Damen und Herren, liebe DG}

dieses Grußwort sende ich aus Washington DC. Es gibt viele Dinge während meines USA-Aufenthalts, die mich an die DGLRM erinnern. Im Jahr 1787 kamen Delegierte aus den 13 ursprünglichen Bundesstaaten zusammen, um die Konföderationsartikel zu überarbeiten. Doch nach einigen Diskussionen zeigte sich, dass eine völlig neue Verfassung nötig war. Auch im Vorstand der DGLRM gab es Überlegungen, die Satzung zu überarbeiten und den aktuellen Gegebenheiten anzupassen. Auch hier zeigte sich, dass eine neue Satzung nötig wurde, da bei diversen Änderungen die Satzung und ihre Änderungen nicht sinnvoll darstellbar sind. Und auch in der DGLRM gibt es Diskussionen über einige Änderungen in der Satzung. Der neue Satzungsentwurf wird Ihnen mit der Einladung zur Vorstandssitzung zugehen und natürlich Thema der Mitgliederversammlung sein. In diesem Zusammenhang möchte ich noch einmal ausdrücklich der Arbeitsgruppe Satzung (bestehend aus Eckard Glaser, Dr. Jörg Hedtmann, PD Dr. Carla Ledderhos, Dr. Matthias von Mülmann und Prof. Dr. Hans Pongratz) für ihre hervorragende Arbeit danken.

Bei meinem Besuch der Vereinten Nationen konnte ich sehen, was gemeinsame

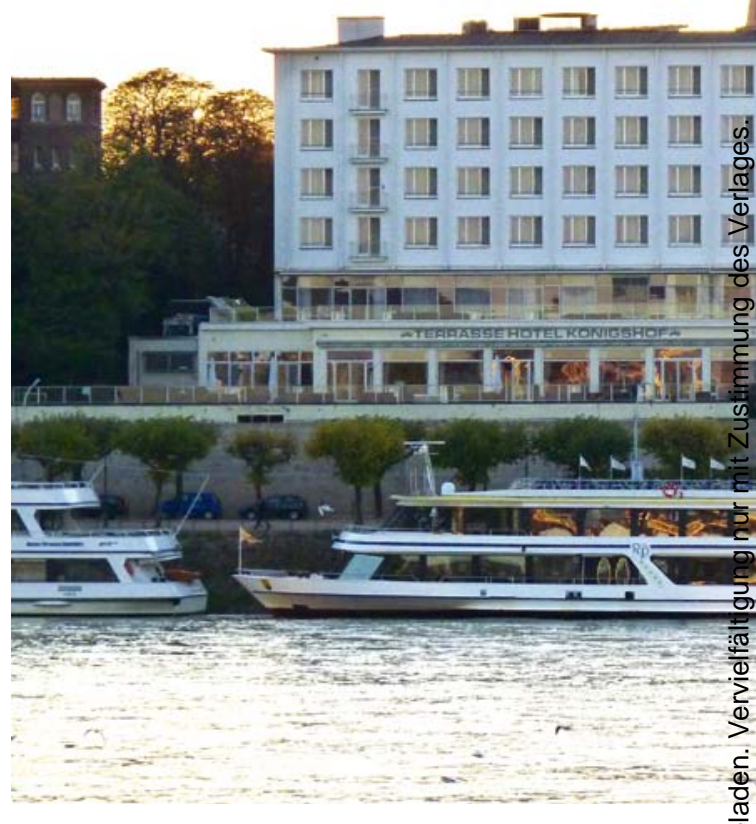

Die 50. Jahrestagung findet im Hotel Königshof in Bonn statt, we

Aktivitäten bewirken können. Auch wenn es häufig schwierig ist, Konsens in der UN zu erreichen (speziell wenn es einige Veto-Mächte gibt), so sind die Erfolge im Bereich Sicherheit, aber vor allem

\title{
Fortbildungen 2012
}

EUSAM $-5^{\text {th }}$ International Aero Medical Examiner Seminar (FAA)

European School of Aviation Medicine

Termin: 26.08.-26.08.2012

Ort: Berlin,

Hotel Palace

Kontakt: Frau Froese, Tel.: +49 (0)69/69691222

Internet: www.flugmed.org/ www.eusam.org

Ausgebucht: EUSAM - Basic course 22 (englisch)

European School of Aviation Medicine

Termin: 01.09.-09.09.2012

Ort: $\quad$ Frankfurt Airport, Lufthansa-Basis

Kontakt: Frau Froese, Tel.: +49 (0)69/69691222

Internet: www.flugmed.org/ www.eusam.org
DAF - Refresher-Seminar Flugmedizin (deutsch)

Deutsche Akademie für Flug- und Reisemedizin

Termin: 26.10.-28.10.2012

Ort: Seeheim-Jugenheim, Lufthansa Training \& Conference Center

Kontakt: Frau Froese, Tel.: +49 (0)69/69691222

Internet: www.flugmed.org

EUSAM - Advanced course 22 (englisch) European School of Aviation Medicine Termin: 08.12.-16.12.2012

Ort: Frankfurt Airport, Lufthansa-Basis

Kontakt: Frau Froese, Tel.: +49 (0)69/69691222

Internet: www.flugmed.org/ www.eusam.org 


\section{LRM-Mitglieder,}

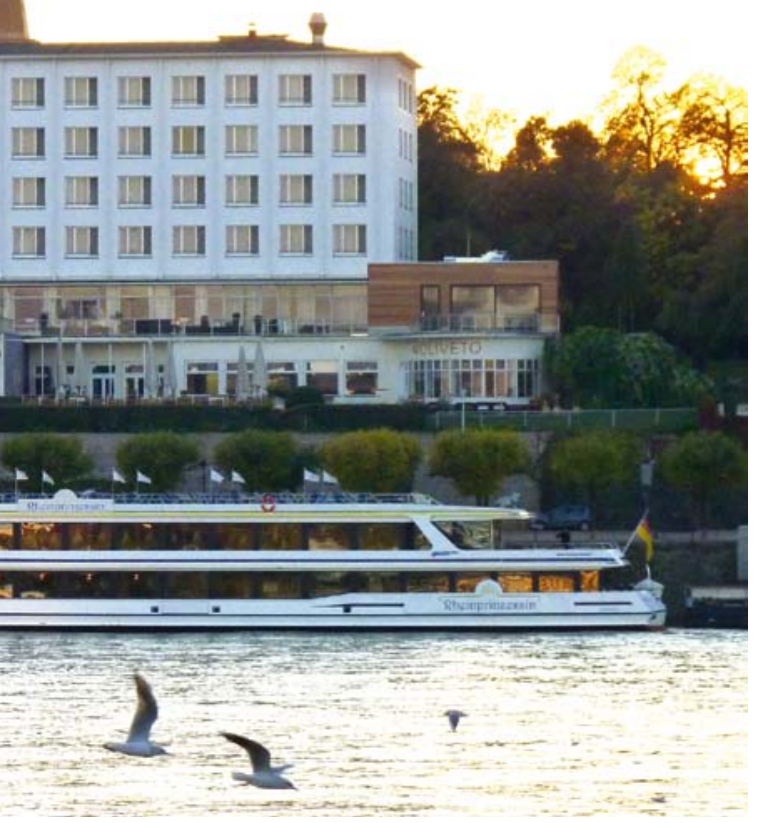

nes sowohl am Rhein liegt, als auch in der Nähe der Innenstadt.

auch Bildung beeindruckend. Ich halte den gemeinsamen Weg in Europa mit der European Society of Aerospace Medicine für den richtigen Weg, um Ziele zu erreichen. In diesem Sinne freue ich mich auch sehr, dass unsere Jahrestagung 2013 zusammen mit den Österreichern und den Schweizern stattfindet. Gemeinsam sind wir stärker und können viel voneinander lernen. Im Gegensatz zu den USA vereint uns in Europa eine ähnliche Geschichte, ähnliche Rechtssysteme, die European Aviation Safety Agency und dass Englisch für die meisten von uns eine Fremdsprache ist. Aus diesen Gründen halte ich es nach wie vor für eine interessante und wichtige Idee, ein europäisches Aerospace Medical Journal zu initiieren.

Ich hatte auch die Gelegenheit bei meinem Aufenthalt in Washington DC die Büros der Aerospace Medical Association in Alexandria zu besuchen. Alexandria ist ein sehr schönes Städtchen mit kleinen Häusern und einer schmucken „Altstadt“, welches per U-Bahn mit Washington DC verbunden ist. Die AsMA hat ihre Büros über 2 Etagen, im Treppenhaus hängen die Bilder aller bisherigen Präsidenten und einige AsMA-Auszeichnungen. Der Executive Director Jeff Sventek arbeitet zusammen mit 5 Damen an dem Erfolg von AsMA. Leider wurde das für 2018 geplante AsMA-Meeting in Europa abgesagt, da die meisten beim Staat beschäftigten amerikanischen AsMA-Mitglieder in der momentan schlechten Finanzlage aus finanziellen Gründen nicht hätten teilnehmen können. Wir haben uns aber darauf verständigt, dass dieses Projekt wieder in Angriff genommen werden sollte, sobald sich die weltweite Finanzkrise entspannt hat.

Ich freue mich riesig auf unsere diesjährige Jahrestagung in Bonn. Für den Festvortrag konnten wir Dr. Richard Williams, den wichtigsten NASA-Mediziner, gewinnen. Er ist für alle nationalen und internationalen NASA-Aktivitäten, bei denen Menschen involviert sind, verantwortlich. Wir hoffen natürlich auf gutes Wetter beim Besuch der Villa Hammerschmidt, da auch eine Führung durch den Garten der Villa geplant ist.

Ich freue mich sehr, Sie im September in Bonn wiederzusehen.

Ihre Claudia Stern
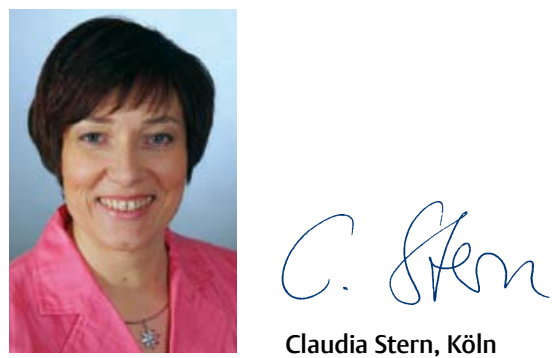

Claudia Stern, Köln

Kongresse 2012

\section{Symposium DGVM/DGVP 2012}

Deutsche Gesellschaft für Verkehrsmedizin und Deutsche Gesellschaft für Verkehrspsychologie

Termin: 07.09.-08.09.2012

Ort: Hamburg

Kontakt: Frau Mandy Wagner, Tel.: +49 (0)3641/3116160

Internet: www.conventus.de/ verkehr2012 $6^{\text {th }}$ International Congress of Aviation and Space Medicine (ICASM)

Int. Academy of Aviation a. Space Medicine Termin: 16.09.-20.09.2012

Ort: Melbourne, Australien

Internet: www.iaasm.org

50. Jahrestagung DGLRM

Dt. Ges. f. Luft- und Raumfahrtmedizin

Termin: 27.09.-29.09.2012
Ort: Bonn, Hotel Königshof

Internet: www.dglrm.de

$3^{\text {rd }}$ European Conference in Aerospace
Medicine (ECAM)
$\begin{array}{ll}\text { Termin: } & \text { 09.11.-11.11.2012 } \\ \text { Ort: } & \text { London, } \\ & \text { Heathrow, } \\ & \text { Sheraton Skyline Hotel }\end{array}$

Internet: www.ecam2012.eu

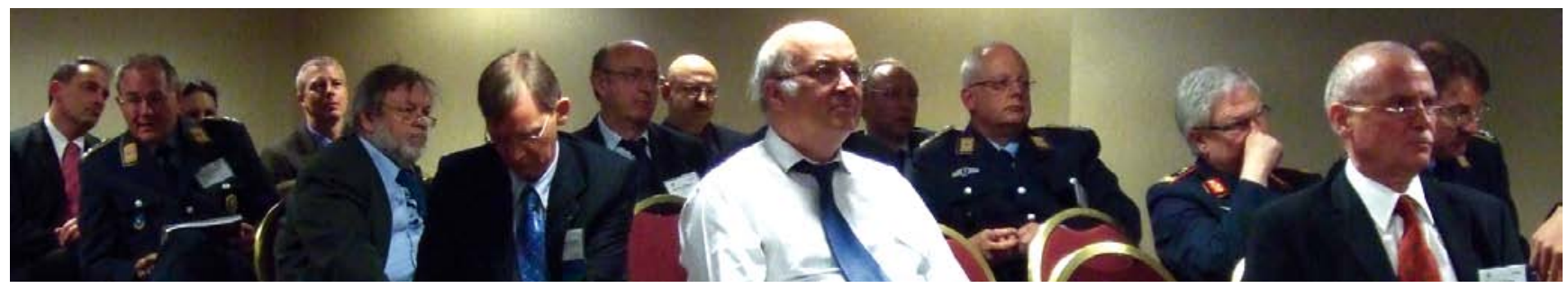

Bei der Tagung der Aerospace Medical Association in Atlanta gab es erstmalig mehr Anmeldungen aus dem Ausland als aus den USA. An der traditionellen Sitzung der DGLRM Montagnachmittag nahmen rund 30 Personen teil, auch aus dem Ausland. 


\title{
Deutsche Gesellschaft für Luft- und Raumfahrtmedizin e. V.
}

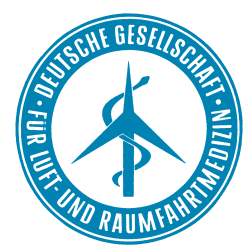

\section{Jahrestagung 27.-29.09.2012 im Hotel Königshof in Bonn}

\author{
Anmeldung \\ per Fax: +49 (0) 2286297331 \\ per Mail: dglrm2012@m4-cp.de
}

Kongressteilnahme 28.09. und 29.09.2012

Teilnahme an der Vorstandsrats-Sitzung am 27.09.2012

Teilnahme an den Fachbesichtigungen am 28.09.2012:

- Hubschrauber-Fliegergruppe der Bundespolizei mit $\square$ Personen oder

- ADAC HEMS Academy mit $\square$ Personen

Teilnahme am Festabend am 29.09.2012 mit $\square$ Personen

(Anmeldung bis 31.08.2012 erforderlich, Sie werden wegen der Sicherheitsüberprüfung durch das Bundespräsidialamt in einem separaten Anschreiben zur namentlichen Anmeldung aufgefordert

- Teilnehmerzahl begrenzt)

Titel, Name, Vorname

Institution / Firma

Anschrift

Telefon / Telefax

E-Mail (unbedingt erforderlich)

Teilnahmegebühren

Anmeldung bis 30.06.2012

Mitglieder

$80,-€$

Anmeldung ab 01.07.2012

Student mit Ausweis 40,-€

Nichtmitglieder

$120,-€$

Mitglieder

$120,-€$

Nichtmitglieder $\quad 160,-€$

Fachbesichtigungen am 28.09.2012: $\quad 10,-€$

Festabend am 29.09.2012: $\quad$ 45,-€

Mitglied der DGLRM $\square$ Nichtmitglied $\quad \square$ Student $\quad \square$ Ich habe Interesse an einer Mitgliedschaft

Ihre Anmeldung wird erst nach Eingang der Teilnahmegebühren gültig.

Hierzu steht Ihnen bei Angabe Ihrer Kontodaten

das Lastschriftverfahren zur Verfügung:

Bitte buchen Sie die Teilnahmegebühr von meinem Konto ab:
BLZ

Bank

Kontoinhaber

Datum

Unterschrift
Alternativ können Sie die

Teilnahmegebühr überweisen an:

DGLRM e. V.

Kontonummer: 388948201

BLZ: 20070000

Deutsche Bank Hamburg

Verwendungszweck:

Jahrestagung 2012 